\title{
MODELOS LINEARES E NÃO LINEARES COM USO DE COVARIANTES PARA RELAÇÃO HIPSOMÉTRICA DE DUAS ESPÉCIES DE PINUS TROPICAIS
}

\author{
LINEAR AND NONLINEAR MODELS WITH USE OF COVARIATES FOR HYPSOMETRIC \\ RELATIONSHIP OF TWO TROPICAL PINE SPECIES
}

\author{
Ayuni Larissa Mendes Sena ${ }^{1}$ Antônio José da Silva Neto ${ }^{2}$ Gabriel Marcos Vieira Oliveira ${ }^{3}$ \\ Natalino Calegario ${ }^{4}$
}

\begin{abstract}
RESUMO
Tradicionalmente, a maior parte dos estudos utiliza modelos lineares para o ajuste da relação hipsométrica, tendo o diâmetro a 1,30 m do solo (DAP) como única variável independente. Entretanto, sabe-se que a linearidade não caracteriza fielmente o crescimento biológico e que outras variáveis relativas ao povoamento, ou às árvores individuais, exercem influência sobre o crescimento em altura. O objetivo deste trabalho foi avaliar o desempenho de modelos lineares e não lineares no ajuste da relação hipsométrica de duas espécies de Pinus tropicais, bem como verificar o efeito da adição de covariantes na estimativa dos parâmetros destes modelos. Os dados utilizados provêm de plantios de Pinus caribaea var. hondurensis e Pinus oocarpa, com diferentes idades e localizados em áreas com diferentes índices de sítio, nos municípios de Nova Ponte e Indianópolis, estado de Minas Gerais. Modelos lineares e não lineares foram ajustados em suas formas originais e com a inclusão das covariáveis idade, sítio e espécie nas estimativas de cada parâmetro dos modelos. Os modelos foram comparados por meio dos critérios estatísticos: erro padrão da estimativa $\left(\mathrm{S}_{\mathrm{yx}}\right)$, Critério de Informação de Akaike (AIC), Critério de Informação Bayesiano (BIC) e também pela análise gráfica de resíduos padronizados. Constatou-se que modelos não lineares demonstram maior capacidade de descrever o comportamento da variável altura, e que a inclusão das covariáveis tornou superior a qualidade dos ajustes, considerando os critérios estatísticos de precisão adotados.
\end{abstract}

Palavras-chave: curvas $\mathrm{h} / \mathrm{d}$; pinus tropicais; relação altura-diâmetro; covariáveis.

\section{ABSTRACT}

Traditionally, most studies use linear models to adjust the hypsometric relationship using diameter at breast height $(\mathrm{DBH})$ as the only independent variable. However, it is known that the linearity does not characterizes faithfully biological growth and other variables related to forest stand, or individual trees, influence in height growth. The aim of this study was to evaluate linear and nonlinear models' performance for hypsometric relationship of two tropical pine species, as well as the effect of covariates adding in models' parameters estimation. The data used provided from Pinus caribaea var. hondurensis and Pinus oocarpa stands, with different ages and site index, located in the counties of Nova Ponte and Indianápolis, Minas Gerais state. Linear and nonlinear models were fitted in its original form and including the covariates age, site index and species in the estimation of each models' parameter. The models were compared using the following statistical criteria: residual standard error (Syx), Akaike Information Criteria (AIC), Bayesian Information Criteria (BIC) and

1 Engenheira Florestal, MSc., Analista Ambiental do Instituto Brasileiro de Meio Ambiente e dos Recursos Naturais Renováveis (IBAMA), SCEN Trecho 2, Edifício Sede, Bloco A, Asa Norte, CEP 70818-900, Brasília (DF), Brasil. ayunisena@gmail.com

2 Engenheiro Florestal, MSc., Analista em Desenvolvimento Regional da Companhia de Desenvolvimento dos Vales do São Francisco e do Parnaíba, SGAN 601, Conj. I, Ed. Dep. Manoel Novaes, Asa Norte, CEP 70830-901, Brasília (DF), Brasil. antoniojsnd@yahoo.com.br

3 Engenheiro Florestal, DSc., Agente em Desenvolvimento Agropecuário (IDAF), Rua Dr. José Coelho dos Santos, 231, Centro, CEP 29400-000, Mimoso do Sul (ES), Brasil. gabrielmvo@gmail.com

4 Engenheiro Florestal, PhD., Professor do Departamento de Ciências Florestais, Universidade Federal de Lavras, Caixa Postal 3037, CEP 37200-000, Lavras (MG), Brasil. calegari@dcf.ufla.br 
also by graphical analysis of standardized residuals. It was found that nonlinear models show greater ability to describe height behavior, and the inclusion of covariates in models' parameters improved its fit quality, considering the statistical accuracy criteria adopted.

Keywords: h/d curves; tropical pines; height-diameter relationship; covariates.

\section{INTRODUÇÃO}

O inventário florestal é uma etapa de extrema importância para as atividades florestais, uma vez que permite obter estimativas suficientemente precisas sobre características qualiquantitativas dos povoamentos, fornecendo subsídios para o manejo dos recursos. Entretanto, esta atividade apresenta, em geral, custos elevados em função do tempo demandado para a coleta de informações e da necessidade de equipes treinadas. Segundo Silva et al. (2007), na operacionalização do inventário o diâmetro a altura do peito (DAP) é uma variável de fácil obtenção, porém, o mesmo não é válido para a altura, sendo comum a ocorrência de erros na mensuração desta variável.

Relação hipsométrica é um artifício utilizado no meio florestal com o objetivo de estimar a altura de árvores, normalmente em função do DAP, a partir do uso de modelos de regressão. De acordo com Bartoszeck et al. (2002), essa é a forma mais econômica de obtenção da variável altura, para todo o povoamento. A partir de um conjunto de alturas de algumas das árvores e os respectivos diâmetros, estabelece-se uma relação de regressão da altura sobre o diâmetro, a qual é empregada para estimar as alturas das demais árvores da parcela em função apenas dos diâmetros já medidos. Neste sentido, modelos estocásticos para a relação hipsométrica são amplamente utilizados para reduzir os custos do inventário.

Diversos autores têm relatado que a altura dos indivíduos é significativamente afetada pela idade, qualidade do sítio florestal, espécie e densidade do plantio (LEITE e ANDRADE, 2003; MACHADO et al., 1994; BARTOSZECK et al., 2004). Barros et al. (2002) fizeram alusão ao aspecto positivo da inclusão dessas variáveis nos modelos hipsométricos, relatando que há melhoria nas medidas de precisão dos ajustes desses modelos, que tendem a ser superiores àqueles obtidos com o ajuste de modelos tradicionais, que utilizam apenas o DAP como variável independente.

Modelos lineares têm sido muito utilizados para a relação hipsométrica, principalmente devido à facilidade de ajuste (FERREIRA, 2009). Contudo, não são os mais adequados para expressar esta relação por acarretar erros de estimativa, já que, muitas vezes, esta relação apresenta-se como não linear (CALEGARIO et al., 2005). Ainda assim, os modelos não lineares têm sido subutilizados em função da dificuldade de ajuste e da necessidade de muitos recursos computacionais para tal. Todavia, diversos autores têm obtido êxito na utilização destes modelos para estimar a altura total de populações florestais, podendo ser destacados os trabalhos de Zhang (1997), Fang e Bailey (1998), Calama e Montero (2004), Calegario et al. (2005), Newton e Amponsah (2007), dentre outros.

Desta forma, este trabalho teve como objetivo avaliar o desempenho de modelos de regressão lineares e não lineares na estimativa da relação hipsométrica de duas espécies de pinus tropicais, bem como o efeito da adição de covariantes nos parâmetros destes modelos.

\section{MATERIAIS E MÉTODOS}

\section{Caracterização da área de estudos}

O estudo foi conduzido na área manejada pela empresa Caxuana Reflorestamento S.A., localizada nos municípios de Nova Ponte e Indianópolis, no estado de Minas Gerais. A região apresenta altitude média de $980 \mathrm{~m}$ e clima tipo Aw, segundo a classificação de Köppen, com temperatura média anual de $22,6^{\circ} \mathrm{C}$ e precipitação média anual de $1.473 \mathrm{~mm}$, concentrada de outubro a março. Os solos são arenosos e argilosos com predominância do latossolo vermelho distrófico (EMBRAPA, 2006). A topografia é de plana a levemente ondulada.

\section{Coleta e análise dos dados}

Os dados são oriundos de inventários realizados em povoamentos puros de Pinus caribaea var. hondurensis (Sénécl.) W.H.G. Barrett \& Golfari e Pinus oocarpa Schiede ex Schltdl. A amostragem foi realizada com base em 462 parcelas de área variável $\left(120 \mathrm{a} 700 \mathrm{~m}^{2}\right)$ alocadas em quatro plantios distintos, onde foram mensurados os diâmetros a $1,30 \mathrm{~m}$ do solo (DAP) de 34.500 árvores e, dentre 
essas, a altura de 15.732 árvores, distribuídas entre os plantios. De acordo com a classificação da qualidade dos sítios florestais, os dados foram distribuídos em cinco classes de sítio para espécie Pinus caribaea var. hondurensis e três classes para Pinus oocarpa, definidas a partir das alturas das árvores dominantes e codominantes médias, considerando o conceito proposto por Assmann (1961). Os índices de sítio variaram de 9,5 a $39,5 \mathrm{~m}$ e de 16,5 a 26,5 $\mathrm{m}$, para Pinus caribaea var. hondurensis e Pinus oocarpa, respectivamente, considerando a idade de referência 12 anos. Os plantios têm idade variando aproximadamente de 2,5 a 16,7 anos.

As análises dos dados foram realizadas através do software estatístico $\mathrm{R} \quad 2.9 .2 \quad$ ( $\mathrm{R}$ DEVELOPMENT CORE TEAM, 2008), com auxílio do pacote $n$ lme.

\section{Modelos avaliados}

Os modelos lineares e não lineares, apresentados na Tabela 1, foram avaliados quanto à qualidade dos seus ajustes, tendo-se verificado o efeito da adição das covariantes sítio, idade e espécie na decomposição dos parâmetros destes modelos, que, nesse caso, foram chamados genericamente de "modelos modificados".

\section{Ajuste e critérios de avaliação dos modelos}

Os modelos lineares e não lineares originais foram ajustados pelo método dos mínimos quadrados ordinários, enquanto que os modelos modificados pela inclusão das covariáveis foram ajustados pelo método dos mínimos quadrados generalizados (MCCULLAGH \& NELDER, 1983), ambos pressupondo $\varepsilon \sim \mathrm{N}\left(0, \sigma^{2}\right)$.

A avaliação dos modelos foi feita por meio da análise gráfica da distribuição dos resíduos padronizados e conforme os indicativos de precisão de ajuste: erro padrão da estimativa $\left(\mathrm{S}_{\mathrm{yx}}\right)$, Critério de Informação de Akaike (AIC) e Critério de Informação Bayesiano (BIC). Nos modelos 2 e 3 (logaritmizados), o erro padrão da estimativa $\left(S_{y x}\right)$ foi recalculado a fim de obter uma mesma base de comparação. O Critério de Informação de Akaike (AIC) e o Critério de Informação Bayesiano (BIC) são testes que permitem uma comparação entre modelos não aninhados e penalizam aqueles com maior número de parâmetros, adequando-os ao conceito de parcimônia. Em ambos, menores valores indicam melhor ajuste (WOLFINGER, 1993).
Erro Padrão da Estimativa $\left(\mathrm{S}_{\mathrm{yx}}\right)$ :

$$
S_{y x}=\sqrt{\frac{\sum_{i=1}^{n}\left(Y_{i}-\hat{Y}_{i}\right)^{2}}{n-p}}
$$

Critério de informação de Akaike (AIC):

$$
A I C=-2 \ln (m v)+2 p
$$

Critério de Informação Bayesiano (BIC):

$$
B I C=-2 \ln (m v)+p \ln (n)
$$

Em que: $Y_{i}$ : altura observada $(\mathrm{m}) ; \hat{Y}_{i}$ : altura estimada pelo modelo $(\mathrm{m}) ; n$ : número de observações; $p$ : número de parâmetros do modelo; $m v$ : máxima verossimilhança.

\section{RESULTADOS E DISCUSSÃO}

As estimativas dos parâmetros e as estatísticas de ajuste para os modelos lineares, em suas formas originais e modificadas, são apresentadas nas Tabelas 2 e 3 , respectivamente. Uma análise comparativa entre as duas tabelas demonstra que a adição das covariantes nos parâmetros referentes ao intercepto e à inclinação resultou em melhoria significativa no ajuste e redução do erro padrão de estimativa, sobretudo dos modelos 1 e 4. Em relação ao modelo 1, a adição de covariantes em comparação com o modelo original proporcionou redução no erro padrão de estimativa $\left(S_{y x}\right)$ de $49,3 \%$ e ganhos de 30 e $36,9 \%$ em relação aos critérios de informação de Akaike (AIC) e Bayesiano (BIC), respectivamente. No caso do modelo 4, houve uma redução expressiva de $63,6 \%$ no $S_{y x}$ e ganhos de 36,8 e $28,9 \%$ em relação aos AIC e BIC, respectivamente. Entretanto, para os modelos logarítmicos 2 e 3, a decomposição dos parâmetros, apesar de significativa, resultou em aumento do erro da estimativa, demonstrando que estes modelos são inadequados para a estimativa da altura quando se considera uma base de dados robusta.

Com relação à distribuição dos resíduos padronizados dos modelos lineares em suas formas originais, pode-se afirmar que, independentemente do modelo considerado, as distribuições residuais se mostraram inadequadas, havendo grande dispersão, desbalanço dos valores e elevada tendenciosidade nas estimativas (Figura 2), provavelmente ocasionado pela alta variabilidade dos dados e 
TABELA1: Modelos de regressão lineares e não lineares em suas formas originais e modificadas com inclusão de covariantes.

TABLE 1: Linear and nonlinear regression models in their original forms and modified by covariates inclusion.

\begin{tabular}{|c|c|c|}
\hline Id/Referência/ Tipo & Forma & Modelo \\
\hline \multirow{2}{*}{$\begin{array}{l}1 \\
\text { Trorey } \\
\text { Linear }\end{array}$} & Orig. & $\hat{h}_{i}=\beta_{0}+\beta_{1} d_{i}+\beta_{2} d_{i}^{2}+\varepsilon_{i}$ \\
\hline & Modif. & $\hat{h}_{i}=\left(\beta_{00}+\beta_{01} S+\beta_{02} t+\beta_{03} Z\right)+\left(\beta_{10}+\beta_{11} S+\beta_{12} t+\beta_{13} Z\right) d_{i}+\beta_{2} d_{i}^{2}+\varepsilon_{i}$ \\
\hline \multirow{2}{*}{$\begin{array}{c}2 \\
\text { Curtis } \\
\text { Linear }\end{array}$} & Orig. & $\ln \hat{h}_{i}=\beta_{0}+\beta_{1} \frac{1}{d_{i}}+\varepsilon_{i}$ \\
\hline & Modif. & $\ln \hat{h}_{i}=\left(\beta_{\infty 0}+\beta_{01} S+\beta_{02} t+\beta_{03} Z\right)+\left(\beta_{10}+\beta_{11} S+\beta_{12} t+\beta_{13} Z\right) \frac{1}{d_{i}}+\varepsilon_{i}$ \\
\hline \multirow{2}{*}{$\begin{array}{c}3 \\
\text { Stoffels } \\
\text { Linear } \\
\end{array}$} & Orig. & $\ln \hat{h_{i}}=\beta_{0}+\beta_{1} \ln d_{i}+\varepsilon_{i}$ \\
\hline & Modif. & $\ln \hat{h}_{i}=\left(\beta_{00}+\beta_{01} S+\beta_{02} t+\beta_{03} Z\right)+\left(\beta_{10}+\beta_{11} S+\beta_{12} t+\beta_{13} Z\right) \ln d_{i}+\varepsilon_{i}$ \\
\hline \multirow{2}{*}{$\begin{array}{l}4 \\
\text { Assmann } \\
\text { Linear }\end{array}$} & Orig. & $\hat{h}_{i}=\beta_{0}+\beta_{1} \frac{1}{d^{2}}+\varepsilon_{i}$ \\
\hline & Modif. & $\hat{h}_{i}=\left(\beta_{\infty 0}+\beta_{01} S+\beta_{02} t+\beta_{03} Z\right)+\left(\beta_{10}+\beta_{11} S+\beta_{12} t+\beta_{13} Z\right) \frac{1}{d^{2}}+\varepsilon_{i}$ \\
\hline \multirow{2}{*}{$\begin{array}{c}5 \\
\text { Logístico } \\
\text { Não linear }\end{array}$} & Orig. & $\hat{h_{i}}=\frac{\phi_{1}}{1+\exp \left(\frac{\phi_{2}-d_{i}}{\phi_{3}}\right)}+\varepsilon_{i}$ \\
\hline & Modif. & $\hat{h}_{i}=\frac{\left(\phi_{10}+\phi_{11} S+\phi_{12} t+\phi_{13} Z\right)}{1+\exp \left[\frac{\left(\phi_{20}+\phi_{21} S+\phi_{22} t+\phi_{23} Z\right)-d_{i}}{\phi_{3}}\right]}+\varepsilon_{i}$ \\
\hline \multirow{2}{*}{$\begin{array}{l}6 \\
\text { Monomolecular } \\
\text { incompleto } \\
\text { Não linear } \\
\end{array}$} & Orig. & $\hat{h}_{i}=\phi_{1}\left[1-\exp \left(\phi_{2} d_{i}\right)\right]+\varepsilon_{i}$ \\
\hline & Modif. & $\hat{h}_{i}=\left(\phi_{10}+\phi_{11} S+\phi_{12} t+\phi_{13} Z\right)\left\{1-\exp \left[\left(\phi_{20}+\phi_{21} S+\phi_{22} t+\phi_{23} Z\right) d_{i}\right]\right\}+\varepsilon_{i}$ \\
\hline \multirow{2}{*}{$\begin{array}{l}7 \\
\text { Michaelis-Menten } \\
\text { Não linear }\end{array}$} & Orig. & $\hat{h}_{i}=\frac{\phi_{1} d_{i}}{\phi_{2}+d_{i}}+\varepsilon_{i}$ \\
\hline & Modif. & $\hat{h}_{i}=\frac{\left(\phi_{10}+\phi_{11} S+\phi_{12} t+\phi_{13} Z\right) d_{i}}{\left(\phi_{20}+\phi_{21} S+\phi_{22} t+\phi_{23} Z\right)+d_{i}}+\varepsilon_{i}$ \\
\hline \multirow{2}{*}{$\begin{array}{c}8 \\
\text { Gompertz } \\
\text { Não linear }\end{array}$} & Orig. & $\hat{h}_{i}=\phi_{1}\left\{\exp \left[-\phi_{2} \exp \left(-\phi_{3} d_{i}\right)\right]\right\}+\varepsilon_{i}$ \\
\hline & Modif. & $\hat{h}_{i}=\left(\phi_{10}+\phi_{11} S+\phi_{12} t+\phi_{13} Z\right)\left\{\exp \left[-\phi_{2} \exp \left(-\left(\phi_{30}+\phi_{31} S+\phi_{32} t+\phi_{33} Z\right) d_{i}\right)\right]\right\}$ \\
\hline
\end{tabular}

Em que: Id: identificação do modelo; $\hat{h}_{i}$ : altura da i-ésima árvore estimada em metros; Orig.: forma original do modelo; Modif.: forma modificada do modelo; $\beta_{i j}$ : parâmetros da regressão linear a serem estimados; $\phi_{i j}$ : parâmetros da regressão não linear a serem estimados; $d_{i}$ : diämetro a $1,30 \mathrm{~m}$ do solo em centímetros; $S$ : índice de sitio estimado em metros; $t$ : idade em anos; $Z$ : variável dummy associada à espécie, sendo $Z=1$ para Pinus caribaea var. hondurensis e $Z=0$ para Pinus oocarpa; $\varepsilon_{i}$ : erro aleatório.

baixa capacidade dos modelos em explicá-la. Por outro lado, houve melhoria considerável na distribuição de resíduos em todos os modelos lineares após a inclusão das covariáveis, sobretudo no modelo 1 , uma vez que apresentou uma melhor distribuição, sendo esta balanceada e com redução da tendenciosidade (Figura 3). O modelo de Assmann em sua forma original (modelo 4) pode ser considerado inadequado para esta base de dados, pois fornece estimativas negativas para a altura total das árvores, o que foi minimizado após a adição das covariantes (Figuras 2 e 3 ).

De maneira geral, verifica-se que as covariantes sítio, idade e espécie, influenciaram positivamente $\mathrm{o}$ intercepto $\mathrm{e}$ a inclinação dos modelos lineares, deslocando a curva hipsométrica 
TABELA 2: Estimativas dos parâmetros e as estatísticas de ajuste para os modelos lineares nas formas originais.

TABLE 2: Parameters estimates and statistics of fit for linear models the in their original form.

\begin{tabular}{ccccccccc}
\hline Modelo & Parâmetro & Est. & EP & $\mathrm{t}_{\mathrm{c}}$ & Valor-p & $\mathrm{S}_{\mathrm{yx}}$ & AIC & BIC \\
\hline \multirow{2}{*}{1} & $\beta_{0}$ & 3,5531 & 0,1122 & 31,7 & $<0,0001$ & & & \\
& $\beta_{1}$ & 0,3570 & 0,0127 & 28,2 & $<0,0001$ & 2,51 & 73550 & 73581 \\
& $\beta_{2}$ & 0,0077 & 0,0003 & 23,5 & $<0,0001$ & & & \\
2 & $\beta_{0}$ & 3,0498 & 0,0061 & 503,8 & $<0,0001$ & & & \\
& $\beta_{1}$ & $-9,6677$ & 0,0721 & $-134,0$ & $<0,0001$ & & & \\
3 & $\beta_{0}$ & 0,0911 & 0,0145 & 6,3 & $<0,0001$ & & & \\
& $\beta_{l}$ & 0,8402 & 0,0055 & 153,6 & $<0,0001$ & $2,63^{1}$ & $2308^{2}$ & $2331^{2}$ \\
& $\beta_{0}$ & 13,6879 & 0,0453 & 302,3 & $<0,0001$ & & & \\
& $\beta_{1}$ & $-411,2731$ & 4,8268 & $-85,2$ & $<0,0001$ & 3,75 & 86258 & 86281 \\
\hline
\end{tabular}

Em que: Est.: valor da estimativa do parâmetro; EP: erro padrão associado aos parâmetros; $\beta_{i}$ : parâmetros da regressão linear; $t_{c}$ : valor calculado da estatística t de Student; Valor-p: probabilidade de não significância; $\mathrm{S}_{\mathrm{yx}}$ : erro padrão da estimativa; AIC: Critério de Informação de Akaike; BIC: Critério de Informação Bayesiano; ${ }^{1}$ : valores recalculados. 2: valores em base logarítmica.

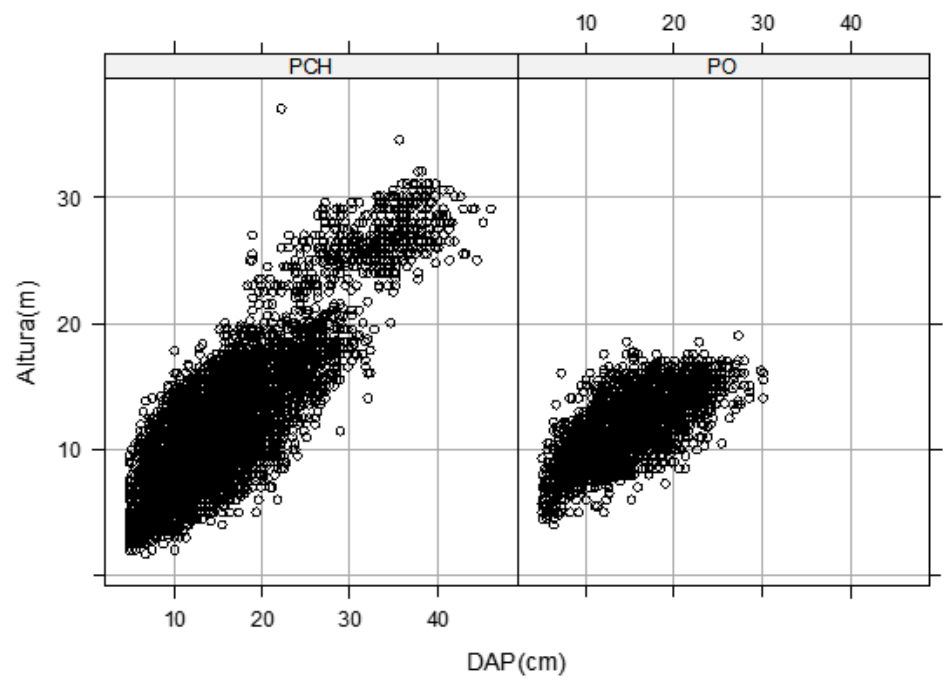

FIGURA 1: Relação entre altura total e o DAP para as duas espécies estudadas, onde PO: Pinus oocarpa; PCH: Pinus caribaea var. hondurensis.

FIGURE 1: Total height and DBH relationship for the two species studied, in which PO: Pinus oocarpa; PCH: Pinus caribaea var. hondurensis.

para cima, considerando os valores positivos dos parâmetros associados aos mesmos, conforme apresentado na Tabela 3.

Dentre os modelos lineares, o modelo 1 foi o que proporcionou melhor ajuste de acordo com o conjunto de indicadores de precisão, sobretudo após a inclusão de covariantes que implicou em melhoria do seu desempenho. A adição de um termo quadrático é uma característica marcante deste modelo que contribuiu com seu 
1

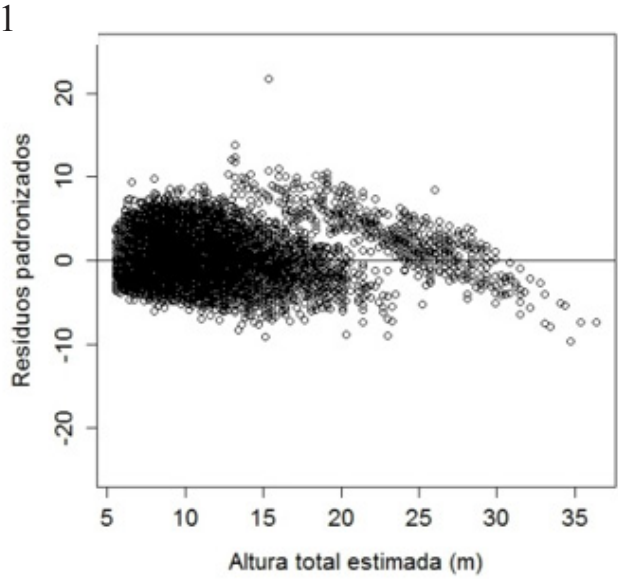

3

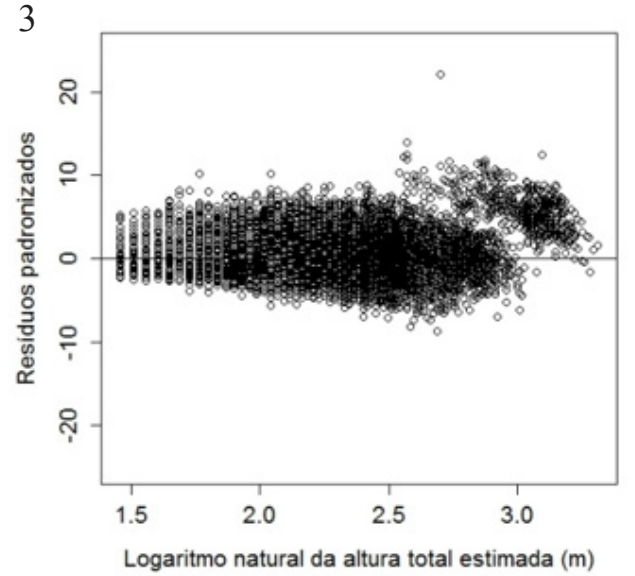

2

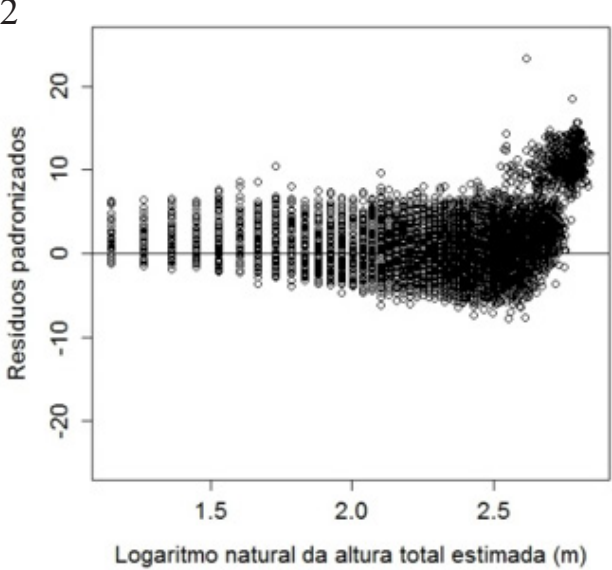

4

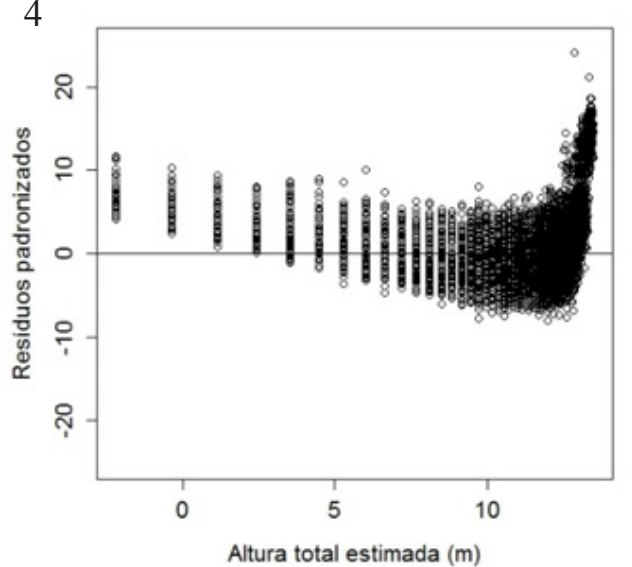

FIGURA 2: Distribuição dos resíduos padronizados dos modelos lineares, em que 1, 2, 3 e 4 referem-se aos modelos de Trorey, Curtis, Stoffels e Assmann, respectivamente.

FIGURE 2: Standardized residuals distribution of linear models, in which 1, 2, 3 and 4 refers to Trorey, Curtis, Stoffels and Assmann models, respectively.

maior desempenho dentre os modelos lineares analisados, visto que contribui para a representação do efeito não linear observado (Figura 1), tornando-o mais representativo do crescimento em altura. Os demais modelos lineares demonstraram baixa capacidade de explicação da variância dos dados, o que pode ser explicado pelo fato de utilizarem apenas a variável independente DAP. Ademais, estes modelos se mostraram inadequados para o ajuste à base de dados não lineares.

Nas Tabelas 4 e 5 são apresentados os resultados das estimativas dos parâmetros e as estatísticas de ajuste para os modelos não lineares em suas formas originais e modificadas, respectivamente. Quando comparados entre si, os quatro modelos não lineares são muito semelhantes em termos de qualidade de ajuste e distribuição dos resíduos, tanto em suas formas originais quanto modificadas. Verifica-se que, em geral, a adição de covariantes melhorou significativamente todos os modelos não lineares utilizados, resultando na redução de $50,6 \%$, em média, do erro padrão da estimativa $\left(S_{y x}\right)$ e melhoria substancial na distribuição dos resíduos, uma vez que estes se apresentaram mais centralizados, balanceados e sem tendências pronunciadas (Figura 5). Verificase também que a inclusão de covariantes promoveu considerável redução nos valores dos critérios AIC e BIC, comparando com os resultados apresentados na Tabela 4, sendo obtidos ganhos de informação médios de $30 \%$ para ambos os critérios, em todos os modelos. Este fato demonstra uma melhoria na qualidade do ajuste e, consequentemente, uma maior explicação da variância total.

Em uma análise comparativa entre os modelos lineares e não lineares, observa-se que 
estes últimos foram ligeiramente superiores, mesmo após a inclusão das covariantes, tendo em vista que apresentaram reduções discretas no erro padrão da estimativa e nos critérios de informação de Akaike e Bayesiano. As estatísticas de desempenho do modelo 1 (Trorey) foram semelhantes àquelas obtidas no

TABELA 3: Estimativas dos parâmetros e as estatísticas de ajuste para os modelos lineares modificados com a inclusão de covariantes.

TABLE 3: Parameters estimates and statistics of fit for linear models modified by the inclusion of covariates.

\begin{tabular}{|c|c|c|c|c|c|c|c|c|}
\hline Modelo & Parâmetro & Est. & EP & $\mathrm{t}_{\mathrm{c}}$ & Valor-p & $S_{y x}$ & AIC & $\mathrm{BIC}$ \\
\hline \multirow{9}{*}{1} & $\beta_{00}$ & $-7,0287$ & 0,1952 & $-36,0$ & $<0,0001$ & \multirow{9}{*}{1,27} & \multirow{9}{*}{52257} & \multirow{9}{*}{52333} \\
\hline & $\beta_{01}$ & 0,2105 & 0,0092 & 22,8 & $<0,0001$ & & & \\
\hline & $\beta_{02}$ & 0,8553 & 0,0191 & 44,7 & $<0,0001$ & & & \\
\hline & $\beta_{03}$ & 1,8813 & 0,0778 & 24,2 & $<0,0001$ & & & \\
\hline & $\beta_{10}$ & 0,2979 & 0,0145 & 20,6 & $<0,0001$ & & & \\
\hline & $\beta_{11}$ & 0,0145 & 0,0007 & 21,1 & $<0,0001$ & & & \\
\hline & $\beta_{12}$ & 0,0144 & 0,0010 & 14,8 & $<0,0001$ & & & \\
\hline & $\beta_{13}$ & $-0,0486$ & 0,0051 & $-9,6$ & $<0,0001$ & & & \\
\hline & $\beta_{2}$ & $-0,0117$ & 0,0004 & $-32,5$ & $<0,0001$ & & & \\
\hline \multirow{8}{*}{2} & $\beta_{00}$ & 1,6579 & 0,0214 & 77,4 & $<0,0001$ & \multirow{8}{*}{$13,85^{1}$} & \multirow{8}{*}{$-16279^{2}$} & \multirow{8}{*}{$-16210^{2}$} \\
\hline & $\beta_{01}$ & 0,0346 & 0,0010 & 33,9 & $<0,0001$ & & & \\
\hline & $\beta_{02}$ & 0,0556 & 0,0012 & 47,9 & $<0,0001$ & & & \\
\hline & $\beta_{03}$ & 0,0470 & 0,0075 & 6,3 & $<0,0001$ & & & \\
\hline & $\beta_{10}$ & $-11,9621$ & 0,2335 & $-51,2$ & $<0,0001$ & & & \\
\hline & $\beta_{l 1}$ & 0,1005 & 0,0107 & 9,4 & $<0,0001$ & & & \\
\hline & $\beta_{12}$ & 0,5923 & 0,0221 & 26,7 & $<0,0001$ & & & \\
\hline & $\beta_{13}$ & 1,2690 & 0,0932 & 13,61 & $<0,0001$ & & & \\
\hline \multirow{8}{*}{3} & $\beta_{00}$ & $-2,5429$ & 0,0519 & $-49,0$ & $<0,0001$ & \multirow{8}{*}{$5,83^{1}$} & \multirow{8}{*}{$-16455^{2}$} & \multirow{8}{*}{$-16386^{2}$} \\
\hline & $\beta_{01}$ & 0,0837 & 0,0025 & 34,0 & $<0,0001$ & & & \\
\hline & $\beta_{02}$ & 0,2490 & 0,0041 & 60,6 & $<0,0001$ & & & \\
\hline & $\beta_{03}$ & 0,5651 & 0,0207 & 27,3 & $<0,0001$ & & & \\
\hline & $\beta_{10}$ & 1,2730 & 0,0204 & 62,3 & $<0,0001$ & & & \\
\hline & $\beta_{11}$ & $-0,0164$ & 0,0010 & $-16,7$ & $<0,0001$ & & & \\
\hline & $\beta_{12}$ & $-0,0572$ & 0,0013 & $-44,4$ & $<0,0001$ & & & \\
\hline & $\beta_{13}$ & $-0,1625$ & 0,0078 & $-20,8$ & $<0,0001$ & & & \\
\hline \multirow{8}{*}{4} & $\beta_{00}$ & $-8,7449$ & 0,1164 & $-75,1$ & $<0,0001$ & \multirow{8}{*}{1,36} & \multirow{8}{*}{54470} & \multirow{8}{*}{54539} \\
\hline & $\beta_{01}$ & 0,5172 & 0,0055 & 94,8 & $<0,0001$ & & & \\
\hline & $\beta_{02}$ & 1,5300 & 0,0063 & 242,5 & $<0,0001$ & & & \\
\hline & $\beta_{03}$ & 1,0074 & 0,0383 & 26,3 & $<0,0001$ & & & \\
\hline & $\beta_{10}$ & 274,5684 & 11,2865 & 24,3 & $<0,0001$ & & & \\
\hline & $\beta_{11}$ & $-11,0147$ & 0,4801 & $-22,9$ & $<0,0001$ & & & \\
\hline & $\beta_{12}$ & $-46,3287$ & 1,3681 & $-33,9$ & $<0,0001$ & & & \\
\hline & $\beta_{13}$ & 24,5501 & 4,5497 & 5,4 & $<0,0001$ & & & \\
\hline
\end{tabular}

Em que: Est.: valor da estimativa do parâmetro; EP: erro padrão associado aos parâmetros $\beta_{i: \text { :: parâmetros da regressão }}$ linear decompostos; $\mathrm{t}$ : valor calculado da estatística t de Student; Valor-p: valor da probabilidade de não significância; $\mathrm{S}_{\mathrm{yx}}$ : erro padrão da estimativa; AIC: critério de informação de Akaike; BIC: critério de informação Bayesiano; ${ }^{1}$ valores recalculados. ${ }^{2}$ valores em base logarítmica. 
1

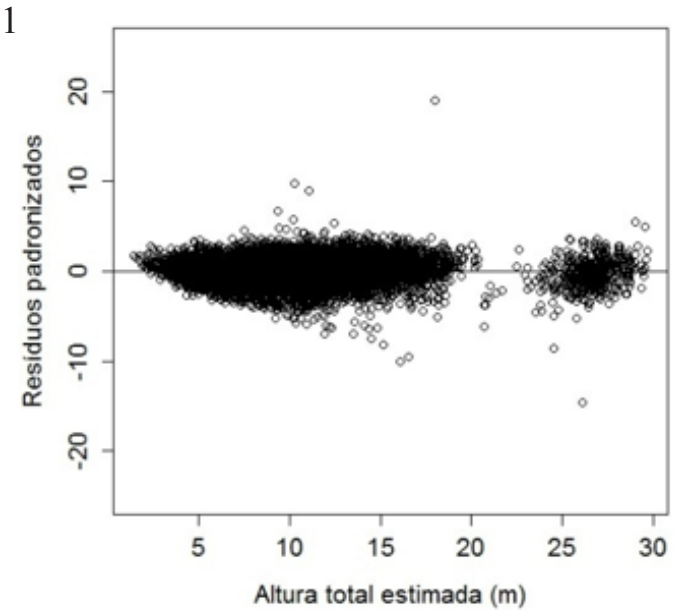

3

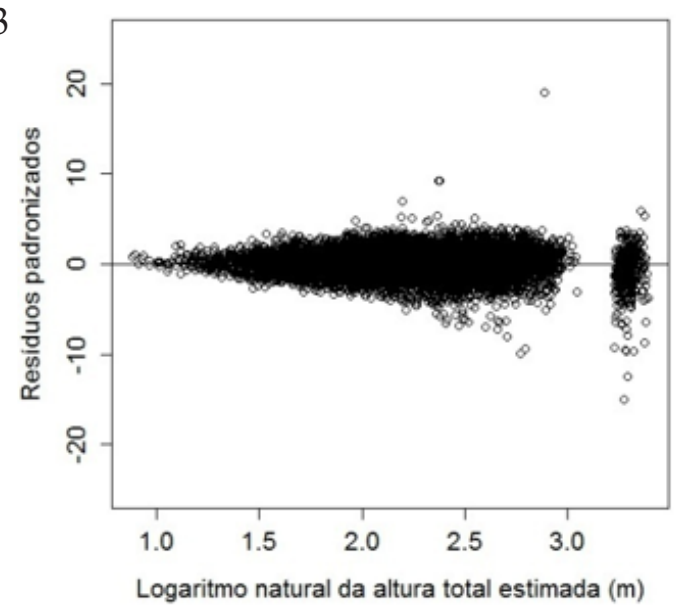

2

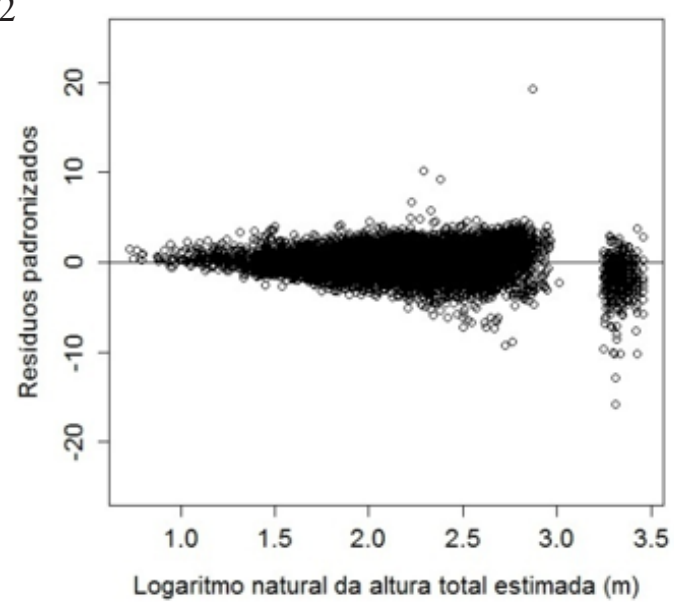

4

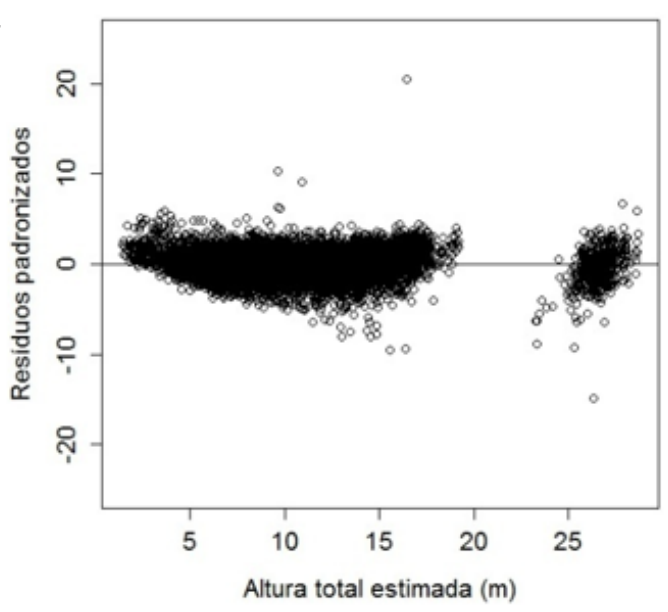

FIGURA 3: Distribuição dos resíduos padronizados dos modelos lineares com adição de covariantes, em que 1, 2, 3 e 4 referem-se aos modelos de Trorey, Curtis, Stoffels e Assmann, respectivamente.

FIGURE 3: Standardized residuals distribution of linear models after covariates addition, in which 1, 2, 3 and 4 refers to Trorey, Curtis, Stoffels and Assmann models, respectively.

TABELA 4: Estimativas dos parâmetros e estatísticas de ajuste para os modelos não lineares nas formas originais.

TABLE 4: Parameters estimates and statistics of fit for nonlinear models in their original form.

\begin{tabular}{ccccccccc}
\hline Modelo & Parâmetro & Est. & EP & $\mathrm{t}_{\mathrm{c}}$ & Valor-p & $\mathrm{S}_{\mathrm{yx}}$ & AIC & BIC \\
\hline \multirow{3}{*}{5} & $\varphi_{1}$ & 45,4977 & 1,1746 & 38,7 & $<0,001$ & & & \\
& $\varphi_{2}$ & 31,4899 & 0,6981 & 45,1 & $<0,001$ & 2,50 & 73494 & 73525 \\
& $\varphi_{3}$ & 13,7758 & 0,1998 & 68,9 & $<0,001$ & & & \\
6 & $\varphi_{1}$ & 176,6000 & 19,2600 & 9,2 & $<0,001$ & & & \\
& $\varphi_{2}$ & $-0,0042$ & 0,0005 & $-8,8$ & $<0,001$ & & 74516 & 74539 \\
7 & $\varphi_{1}$ & 327,6900 & 35,4100 & 9,2 & $<0,001$ & & & \\
& $\varphi_{2}$ & 437,4700 & 49,3700 & 8,9 & $<0,001$ & & 74512 & 74535 \\
& $\varphi_{1}$ & 97,3800 & 6,9460 & 14,0 & $<0,001$ & & & \\
& $\varphi_{2}$ & 3,2330 & 0,0591 & 54,7 & $<0,001$ & 2,50 & 73529 & 73560 \\
& $\varphi_{3}$ & 0,0252 & 0,0010 & 26,1 & $<0,001$ & & & \\
\hline
\end{tabular}

Em que: Est.: valor da estimativa do parâmetro; EP: erro padrão associado aos parâmetros $\varphi_{i}$ : parâmetros da regressão não linear; $t_{c}$ : valor calculado da estatística $t$ de Student ; Valor-p: probabilidade de não significância; $\mathrm{S}_{\mathrm{yx}}$ : erro padrão da estimativa; AIC: critério de informação de Akaike; BIC: critério de informação Bayesiano. 
5

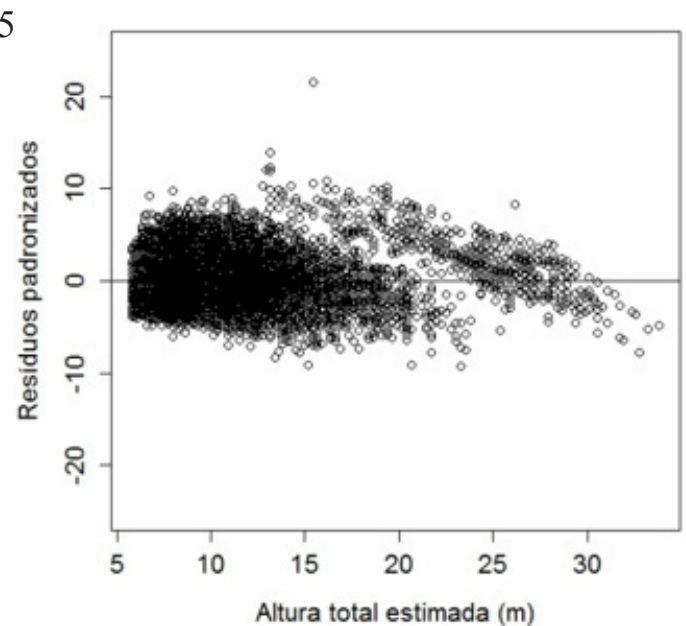

7

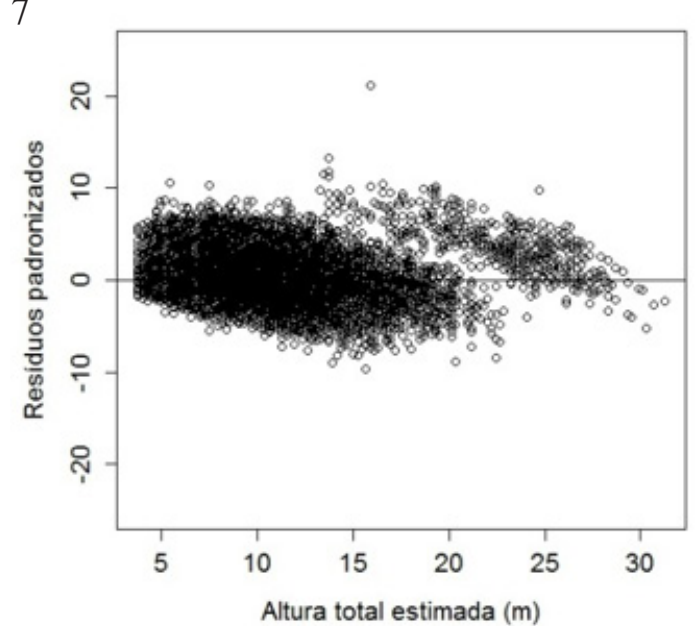

6

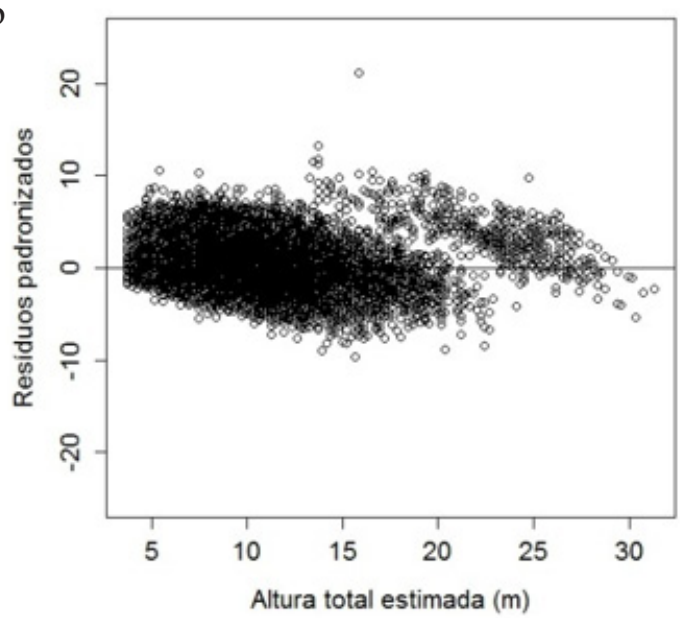

8

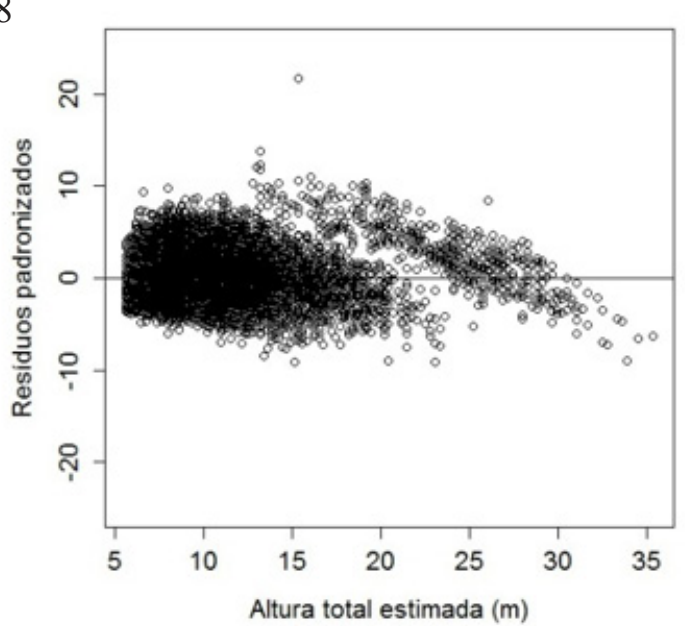

FIGURA 4: Distribuição dos resíduos padronizados dos modelos não lineares nas formas originais, sendo que 5, 6, 7 e 8 referem-se ao modelo Logístico, Monomolecular, Michaelis-Menten e Gompertz, respectivamente.

FIGURE 4: Standardized residuals distribution of nonlinear models in original form, in which 5, 6, 7 and 8 refers to Logistic, Monomolecular, Michaelis-Menten and Gompertz models, respectively.

ajuste dos modelos 5 (Logístico) e 8 (Gompertz), tanto em sua forma original quanto modificada. Conforme exposto, o termo quadrático existente no modelo 1 implica em uma maior capacidade de explicar comportamentos não lineares, quando comparado aos outros modelos lineares avaliados, tornando sua performance semelhante à dos modelos não lineares. Contudo, é importante destacar que, ainda que o ajuste de modelos lineares seja mais simples, recomendam-se os modelos não lineares, pois possuem maior capacidade de explicar a relação não linear observada entre altura e diâmetro das árvores (Figura 1) e, principalmente, pela possibilidade de interpretação biológica dos seus parâmetros.

As covariantes sítio, idade e espécie apresentaram alta influência nos parâmetros dos modelos lineares e não lineares, promovendo melhoria na precisão dos modelos ajustados. Este resultado mostra-se coerente com as afirmações de Leite e Andrade (2003) e Machado et al. (1994), de que estas variáveis são também determinantes na estimativa da altura total do povoamento. Todavia, o efeito da covariante espécie não foi significativo na assíntota dos modelos 6,7 e 8 , de modo que os modelos foram reprocessados excluindo-se os respectivos parâmetros, que por isso não são apresentados na Tabela 5. Calegario et al. (2005) mencionam que a grande flexibilidade do método de decomposição dos parâmetros está no fato de que as variáveis podem estar associadas a um parâmetro e não a outro, dependendo da sua significância. 
Como consequência desse fato, verifica-se que estes modelos não foram capazes de diferenciar a altura máxima estimada para as duas espécies do estudo.

Dentre todos os modelos, podemse recomendar os modelos 5 (Logístico) e 8
(Gompertz) para estabelecimento da relação hipsométrica, sobretudo pela melhor distribuição de resíduos e possibilidade de interpretação biológica dos parâmetros, o que, segundo Calegario et al. (2005) e Guimarães et al. (2009), facilita a escolha

TABELA 5: Estimativas dos parâmetros e estatísticas de ajuste para os modelos não lineares modificados com a inclusão de covariantes.

TABLE 5: Parameters estimates and statistics of fit for nonlinear models modified by the inclusion of covariates.

\begin{tabular}{|c|c|c|c|c|c|c|c|c|}
\hline Modelo & Parâmetro & Est. & $\mathrm{EP}$ & $t_{c}$ & Valor-p & $S_{y x}$ & $\mathrm{AIC}$ & BIC \\
\hline \multirow{9}{*}{5} & $\varphi_{10}$ & $-5,8783$ & 0,2168 & $-27,1$ & $<0,001$ & \multirow{9}{*}{1,27} & \multirow{9}{*}{52102} & \multirow{9}{*}{52178} \\
\hline & $\varphi_{11}$ & 0,4690 & 0,0086 & 54,4 & $<0,001$ & & & \\
\hline & $\varphi_{12}$ & 1,4051 & 0,0085 & 164,9 & $<0,001$ & & & \\
\hline & $\varphi_{13}$ & 0,5538 & 0,0553 & 10,0 & $<0,001$ & & & \\
\hline & $\varphi_{20}$ & 8,3103 & 0,4616 & 18,0 & $<0,001$ & & & \\
\hline & $\varphi_{21}$ & $-0,1398$ & 0,0170 & $-8,2$ & $<0,001$ & & & \\
\hline & $\varphi_{22}$ & 0,1639 & 0,0297 & 5,5 & $<0,001$ & & & \\
\hline & $\varphi_{23}$ & $-2,3329$ & 0,1367 & $-17,1$ & $<0,001$ & & & \\
\hline & $\varphi_{3}$ & 5,5086 & 0,1030 & 53,5 & $<0,001$ & & & \\
\hline \multirow{7}{*}{6} & $\varphi_{10}$ & $-6,2738$ & 0,2422 & $-25,9$ & $<0,001$ & \multirow{7}{*}{1,24} & \multirow{7}{*}{51391} & \multirow{7}{*}{51452} \\
\hline & $\varphi_{11}$ & 0,3952 & 0,0098 & 40,3 & $<0,001$ & & & \\
\hline & $\varphi_{12}$ & 1,8111 & 0,0119 & 152,2 & $<0,001$ & & & \\
\hline & $\varphi_{20}$ & $-0,0696$ & 0,0044 & $-15,9$ & $<0,001$ & & & \\
\hline & $\varphi_{21}$ & $-0,0033$ & 0,0002 & $-16,3$ & $<0,001$ & & & \\
\hline & $\varphi_{22}$ & 0,0056 & 0,0001 & 55,0 & $<0,001$ & & & \\
\hline & $\varphi_{23}$ & $-0,0311$ & 0,0008 & $-37,8$ & $<0,001$ & & & \\
\hline \multirow{7}{*}{7} & $\varphi_{10}$ & $-8,2486$ & 0,4923 & $-16,7$ & $<0,001$ & \multirow{7}{*}{1,28} & \multirow{7}{*}{52351} & \multirow{7}{*}{52412} \\
\hline & $\varphi_{11}$ & 0,5956 & 0,0184 & 32,3 & $<0,001$ & & & \\
\hline & $\varphi_{12}$ & 2,1310 & 0,0450 & 47,4 & $<0,001$ & & & \\
\hline & $\varphi_{20}$ & 10,9679 & 0,6878 & 15,9 & $<0,001$ & & & \\
\hline & $\varphi_{21}$ & $-0,1689$ & 0,0222 & $-7,6$ & $<0,001$ & & & \\
\hline & $\varphi_{22}$ & 0,4751 & 0,0557 & 8,5 & $<0,001$ & & & \\
\hline & $\varphi_{23}$ & $-2,7245$ & 0,0553 & $-49,3$ & $<0,001$ & & & \\
\hline \multirow{8}{*}{8} & $\varphi_{10}$ & $-6,2958$ & 0,2214 & $-28,4$ & $<0,001$ & \multirow{8}{*}{1,24} & \multirow{8}{*}{51407} & \multirow{8}{*}{51484} \\
\hline & $\varphi_{11}$ & 0,3907 & 0,0089 & 43,8 & $<0,001$ & & & \\
\hline & $\varphi_{12}$ & 1,7436 & 0,0108 & 160,7 & $<0,001$ & & & \\
\hline & $\varphi_{2}$ & 1,8033 & 0,0331 & 54,5 & $<0,001$ & & & \\
\hline & $\varphi_{30}$ & 0,1087 & 0,0067 & 16,3 & $<0,001$ & & & \\
\hline & $\varphi_{31}$ & 0,0044 & 0,0003 & 16,0 & $<0,001$ & & & \\
\hline & $\varphi_{32}$ & $-0,0081$ & 0,0002 & $-41,6$ & $<0,001$ & & & \\
\hline & $\varphi_{33}$ & 0,0452 & 0,0013 & 34,5 & $<0,001$ & & & \\
\hline
\end{tabular}

Em que: Est.: valor da estimativa do parâmetro; EP: erro padrão associado aos parâmetros $\varphi_{i::}$ : parâmetros decompostos da regressão não linear; $t$ : valor calculado da estatística $t$ de Student; Valor-p: probabilidade de não significância; $\mathrm{S}_{\mathrm{yx}}$ : erro padrão da estimativa; AIC: critério de informação de Akaike; BIC: critério de informação Bayesiano. Obs.: os parâmetros não significativos das covariantes associadas foram excluídos e o ajuste reprocessado. 
5

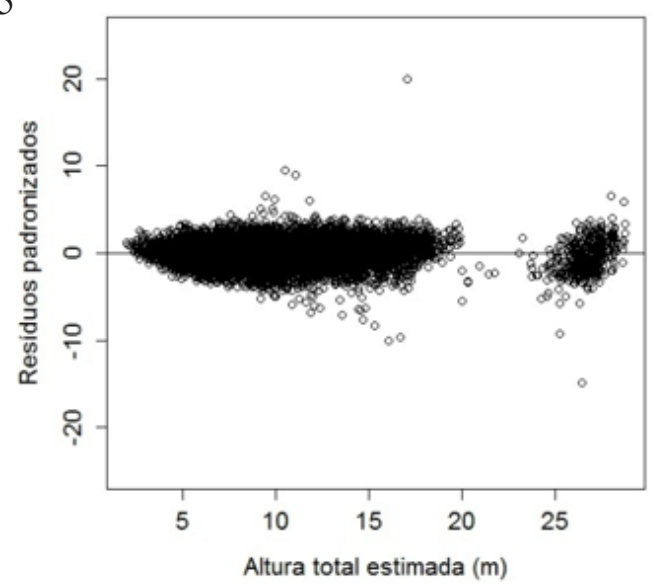

7

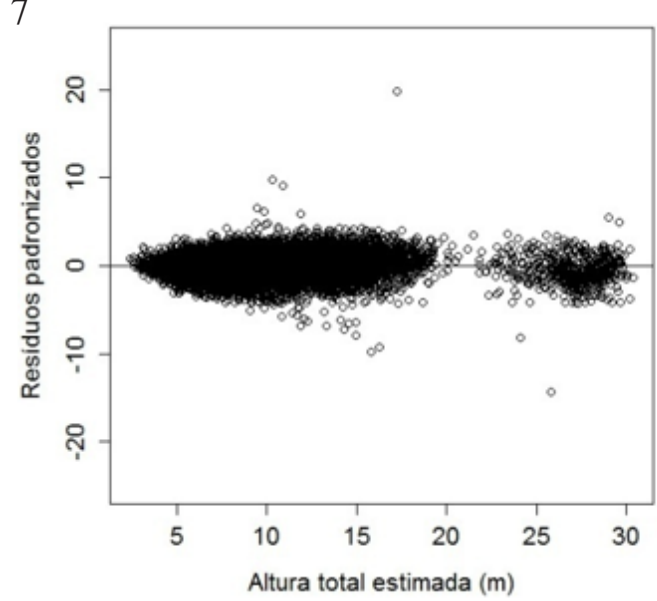

6

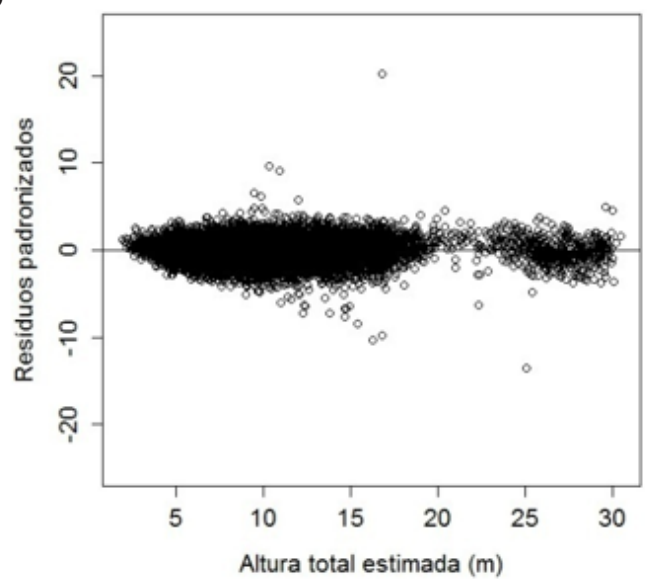

8

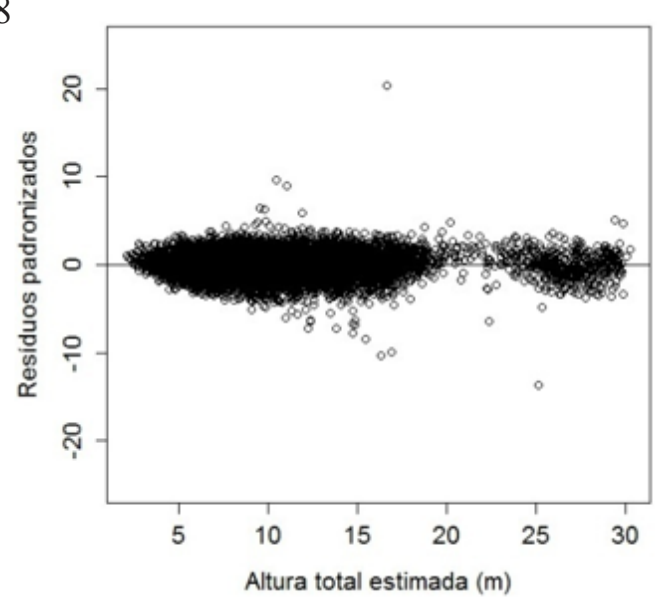

FIGURA 5: Distribuição dos resíduos padronizados dos modelos não lineares nas formas modificadas, em que, 5, 6, 7 e 8 referem-se ao modelo Logístico, Monomolecular, Michaelis-Menten e Gompertz, respectivamente.

FIGURE 5: Standardized residuals distribution of nonlinear models in modified form, in which 5, 6, 7 and 8 refer to Logistic, Monomolecular, Michaelis-Menten and Gompertz models, respectively.

dos valores dos parâmetros iniciais nos processos de convergência para ajuste dos modelos. Fang e Bailey (1998) também fazem recomendações semelhantes ao avaliarem modelos lineares e não lineares para a relação hipsométrica em florestas tropicais, enfatizando a superioridade dos modelos não lineares para este fim. Estes dois modelos apresentam boa capacidade de representação de dados não lineares assintóticos o que pode estar associada ao seu bom desempenho neste estudo de caso.

O modelo linear de Trorey (modelo 1) também se mostrou como uma opção viável para o ajuste da relação hipsométrica, considerando sua elevada performance em relação aos demais modelos lineares avaliados, principalmente após a inclusão das covariantes relacionadas ao povoamento e à espécie. Entretanto, seu uso em detrimento dos modelos não lineares deverá considerar o balanço entre a vantagem da facilidade de ajuste e a desvantagem quanto à impossibilidade de interpretação biológica dos parâmetros e à menor fidelidade à comportamentos não lineares.

\section{CONCLUSÕES}

Os modelos não lineares podem ser utilizados em detrimento de modelos lineares na estimativa da altura em função do diâmetro a altura do peito, tendo em vista que representam com mais fidelidade os padrões biológicos de crescimento com erro próximo ao dos modelos lineares. 
A adição das covariantes idade, índice de sítio e espécie afetam significativamente a relação hipsométrica e devem ser incluídas nos modelos, uma vez que resultam em melhor explicação da variação da altura no povoamento.

Os modelos não lineares Logístico e Gompertz e o modelo linear de Trorey são os mais indicados para estabelecimento da relação hipsométrica, em função da melhor distribuição residual e melhor qualidade de ajuste.

\section{AGRADECIMENTOS}

Agradecemos à Caxuana Reflorestamento S.A. pelo fornecimento da base de dados utilizada neste estudo, a CAPES e ao CNPq pela concessão de bolsas de Mestrado aos três primeiros autores.

\section{REFERÊNCIAS BILIOGRÁFICAS}

ASSMANN, E. Waldertragskunde. München: BLV, Verlagsgessellschaft, 1961. 435p.

BARROS, D.A. de. et al. Comportamento de modelos hipsométricos tradicionais e genéricos para plantações de Pinus oocarpa em diferentes tratamentos. Boletim de Pesquisa Florestal, Colombo,n.45, p.3-28, jul./dez. 2002.

BARTOSZECK, A. C. de P e S. et al. Modelagem da relação hipsométrica para bracatingais da região metropolitana de Curitiba - PR. Floresta, Curitiba, v.32, n.2, p. 189-204, abr./jun., 2002.

BARTOSZECK, A.C. de P e S. et al. Dinâmica da relação hipsométrica em função da idade, do sítio e da densidade inicial de povoamentos de bracatinga na região metropolitana de Curitiba PR. Revista Árvore, Viçosa, v. 28, n. 4, p. 517-533, jul./ago. 2004.

CALAMA, R.; MONTERO, G. Interregional nonlinear height-diameter model with random coefficients for stone pine in Spain. Canadian Journal of Forest Research, Ottawa, v. 34, p. 150-163, jan.2004.

CALEGARIO,N. et al. Melhoria da qualidade de ajuste de modelos biométricos florestais pelo emprego da teoria dos modelos não-lineares generalizados.ScientiaForestalis, Piracicaba, v. 69, p. 38-50, dez., 2005.

EMBRAPA. Sistema brasileiro de classificação de solos. $2^{\text {a }}$ Ed. Rio de Janeiro: Embrapa Solos, 2006.
$306 p$.

FANG， Z.; BAILEY, R.L. Height-diameter models for tropical forests on Hainan Island in southern China, Forest Ecology and Management, Amsterdam, v. 110, p. 315-327, out. 1998.

FERREIRA, D. F. Estatística básica. Lavras: Editora UFLA, $2^{\text {a }}$ Ed. 2009. 664p.

GUIMARÃES, M. A. M. et al. Heigthdiameter models in forestry with inclusion of covariates. Cerne, Lavras, v. 15, n. 3, p. 313-321, jul./set. 2009.

LEITE, H.G.; ANDRADE, V.C. de L.; Importância das variáveis altura dominante e altura total em equações hipsométricas e volumétricas. Revista Árvore, Viçosa, v. 27, n. 3, p. 301-310, mai.jun.2003.

MACHADO, S.A.et al. Análise do comportamento da relação hipsométrica com respeito à idade para plantações de Pinus elliottii no estado do Paraná. Cerne, Lavras, v. 1, n. 1, p. 5-12, 1994.

MCCULLAGH, P.; NELDER, J. A. Generalized linear models. London: Chapman \& Hall, 1983. 261p.

NEWTON, P.F.; AMPONSAH, I.G. Comparative evaluation of five height-diameter models developed for black spruce and jack pine stand-types in terms of goodness-of-fit, lack-of-fit and predictive ability. Forest Ecology and Management, Amsterdam, v.247, p. 149-166, ago. 2007.

R DEVELOPMENT CORE TEAM.R: A language and environment for statistical computing. $R$ Foundation for StatisticalComputing, Vienna, Austria. ISBN 3-900051-07-0. 2008. Disponível em: < (http://www.R-project.org) >. Acesso em: 25 de Janeiro de 2010.

SILVA, G. F.et al. Análise da influência de diferentes tamanhos e composições de amostras no ajuste de uma relação hipsométrica para Eucalyptusgrandis. Revista Árvore, Viçosa, v.31, n.4, p.685-694, jul./ago. 2007.

WOLFINGER, R. Covariance structure selection in general mixed models. Communications in Statistics - Simulation, Ontario, v. 22, n. 4, p. 1079-1106, 1993.

ZAHNG, L. Cross-validation of non-linear growth functions for modeling tree height-diameter relationships. Annals of Botany, London, v. 79(3), p. 251-257, mar. 1997. 